EESTI NSV TEADUSTE AKADEEMIA TOIMETISED.

FOOSIKA * MATEMAATIKA

ИЗВЕСТИЯ АКАДЕМИИ НАУК ЭСТОНСКОИ ССР. ФИЗИКА - МАТЕМАТИКА PROCEEDINGS OF THE ACADEMY OF SCIENCES OF THE ESTONIAN SSR. PHYSICS * MATHEMATICS

$1987,36,2$

\title{
О МАТЕМАТИЧЕСКИХ МОДЕЛЯХ ОПИСАНИЯ РАСПРОСТРАНЕНИЯ ИМПУЛЬСА В НЕРВНОМ ВОЛОКНЕ
}

\author{
(Представил Н. Алумяэ)
}

В данной работе рассматриваются сравнительные свойства некоторых математических моделей, описывающих распространение импульса в нервном волокне. Общеизвестной моделью является модель ФитцХью-Нагумо (ФХН), которая позволяет определить постоянный профиль импульса. как решение обыкновенного дифференциального уравнения третьего порядка $\left[{ }^{1,2}\right]$. Недостатком этой модели является даже на умеренных интервалах времени очень высокая точность коэффициента при третьей производной, что в физическом смысле равносильно высокой точности определения скорости распространения импульса $\left[{ }^{1}\right]$.

Известна и другая математическая модель описания постоянного профиля в виде дифференциального уравнения второго порядка, которое вытекает из соответствующего эволюционного уравнения $\left[{ }^{3,4}\right]$. Решение этого уравнения также качественно правильно описывает распространение нервного импульса, но при этом не требуется такой высокой точности определения параметров задачи.

В данной работе проводится сравнительный анализ этих двух моделей. Показывается, что выбором параметров можно добиться и количественной близости решений двух моделей на промежутке времени, где решение модели ФХН еще адекватно описывает физический процесс. Дается метод для определения порогового значения, которое разделяет области усиления и затухания в терминах фазовой плоскости.

1. Как известно, феноменологическая модель Ходжкина-Хаксли $\left[{ }^{2,5}\right]$, описывающая распространение импульса в нервном волокне, является довольно громоздкой, поэтому во многих случаях пользуются упрощенной моделью ФитцХью-Нагумо $\left[{ }^{2,6-8}\right]$. В обеих моделях исходными являются телеграфные уравнения, в которых пренебрежено индуктивностью. В результате такого приема окончательное уравнение приобретает параболический вид, и решение типа бегущей волны возможно только благодаря наличию дополнительного ионного тока. В случае модели Ходжкина-Хаксли ионный ток аппроксимируется сложным нелинейным выражением, позволяющим довольно точно моделировать поведение токов калиевых и натриевых ионов $\left[{ }^{2,5}\right]$, в случае модели

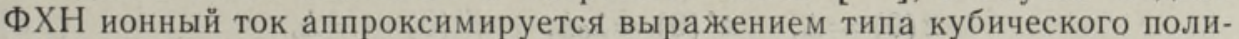
нома $\left[{ }^{1}\right]$. Несмотря на свою относительную простоту, модель ФХН считается вполне работоспособной $\left[{ }^{2,6-8}\right]$.

Однако при расчетах по модели ФХН возникают серьезные проблемы ее адекватности на больших временных интервалах. Рассмотрим эти проблемы детально в свете оригинальных результатов Нагумо и соавторов $\left[{ }^{1}\right]$.

Основная система уравнений модели ФХН, включая уже неполные 
(б̋̉eз индуктивности) телеграфные урав̈нения и специальный ионный ток $J$, имеет вид ([ $\left.{ }^{1}\right]$, система (17))

$$
\begin{gathered}
h \frac{\partial^{2} u}{\partial s^{2}}=\frac{1}{c_{1}} \frac{\partial u}{\partial t}-J \\
J=W+\left(u-1 / 3 u^{3}\right), \\
c_{1} \frac{\partial w}{\partial t}+b=a-u,
\end{gathered}
$$

где $u=u(s, t)-$ напряжение (потенциал). Далее принимается $b=0$, тогда $1<a<2$. Кроме того, принимается $h>0, c_{1}>0$. Отметим, что здесь сохранены обозначения Нагумо [ $\left.{ }^{1}\right]$, и параметр $c_{1}$ в данном случае не имеет размерности скорости. После перехода к новым переменным систему (1.1)-(1.3) можно переписать в виде одного уравнения

$$
\frac{\partial^{3} z}{\partial t \partial x^{2}}=\frac{\partial^{2} z}{\partial t^{2}}+\mu\left(1-z+\varepsilon z^{2}\right) \frac{\partial z}{\partial t}+z,
$$

где $\quad \mu=c_{1}\left(a^{2}-1\right)>0, \quad \varepsilon=\frac{1}{4}\left(a^{2}-1\right) a^{-2}, \quad z=2 a\left(a^{2}-1\right)^{-1}(a-u)$,

$$
x=s\left(c_{1} h\right)^{-1 / 2} .
$$

Уравнения (1.4) решается при удобно выбранных начальных и краевых условиях, задавая при этом начальное распределение потенциала

$$
z(0, t)=z_{0}(t) .
$$

Численный эксперимент $\left[{ }^{1}\right]$ позволяет выяснить наличие порога и асимптотического значения амплитуды импульса в активном волокне. В ходе распространения формируется импульс постоянного профиля. Соответствующее уравнение, описывающее форму постоянного профиля, вытекает из (1.4), если положить

$$
z(\tau)=z(x, t), \quad \tau=t-x \Theta_{N}^{-1}
$$

где $\Theta_{N}-$ постоянная в качестве скорости. Теперь уравнение (1.4) преобразуется к виду

$$
\beta z^{\prime \prime \prime}-z^{\prime \prime}-\mu\left(1-z+\varepsilon z^{2}\right) z^{\prime}-z=0,
$$

где $(\ldots)^{\prime}=\frac{\partial}{\partial \tau}, \beta=\Theta_{N}^{-2}>0$. Нахождение решения $z(\tau)$ уравнения $(1.7)$ включает в себя и описание неизвестного коэффициента $\beta$. Доказано существование такого значения $\beta$, что соответствующее решение уравнения (1.7) приближается к нулю при $\tau \rightarrow \pm \infty\left[{ }^{9-12}\right]$. Необходимо подчеркнуть, что при этом предполагается более медленная динамика переменной $w$ по сравнению с динамикой переменной $u$, т. е. $1 / c_{1} \ll 1$.

Нагумо и др. $\left[{ }^{1}\right]$ предлагают решить уравнение (1.7) при начальных условиях

$$
z(0)=\Delta, \quad z^{\prime}(0)=\lambda_{0} \Delta, \quad z^{\prime \prime}(0)=\lambda_{0}^{2} \Delta,
$$

где $\Delta>0$ совпадает с шагом временной дискретизации, а $\lambda_{0}-$ положительный корень линеаризированного характеристического уравнения. Нами повторен их расчет с более высокой точностью для тех же значений параметров $\mu=3, \varepsilon=0,1, \Delta=0,01$. Результаты расчета, полученные стандартным методом Рунге-Кутта, представлены на рис. 1. Как отмечено уже раньше $\left[{ }^{1}\right]$, решение при больших $\tau$ сильно зависит от 


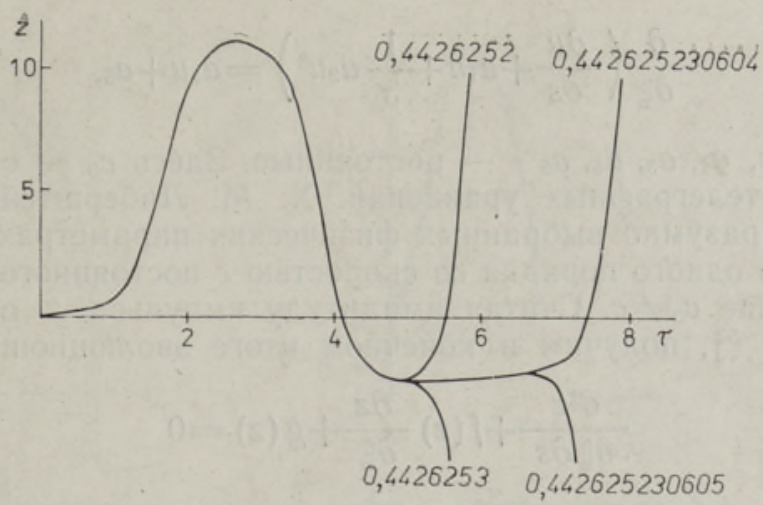

Рис. 1. Профиль импульса, определенный по модели ФХН. Числа - значение $\beta$.

значения $\beta$. На рис. 1 приведены кривые, для которых $\beta$ определяется с точностью: а) 7 знаков; б) 12 знаков. В обоих случаях решение неустойчиво для больших $\tau$, т. е. не удается достичь равновесного состояния. Такого вида неустойчивость на всей временной оси часто свойственна как решениям дифференциальных уравнений, так и многим другим моделям математической физики. Например, преобразование Хенона $\left[{ }^{13}\right]$ имеет неподвижную точку с иррациональными координатами и сколь угодно малая неточность в координатах начального положения не позволяет остаться в окрестности этой точки.

В случае модели ФХН описание физического процесса (напряжения $u(s, t))$ с помощью уравнения (1.7) требует даже на умеренном промежутке времени нереальной точности неизвестного параметра $\beta$. Имея в своем распоряжении модель (1.7) с заданными другими параметрами, нельзя без скрупулезных вычислений определить промежуток времени, на котором модель адекватна. Преодоление этой трудности в рамках данной модели нам представляется невозможным.

2. Менее известен другой подход к построению модели распространения импульса в нервном волокне, предложенный в $\left[{ }^{3,4}\right]$. Как и в модели $Ф Х Н$, основой служат здесь телеграфные уравнения и уравнение ионного тока. В отличие от модели ФХН (а также от модели Ходжкина-Хаксли) телеграфные уравнения используются в полном виде с сохранением индуктивности. Полная система, в которой ионный ток $J$ аппроксимируется тем же кубическим полиномом, как и в $\left[{ }^{1}\right]$, имеет вид

$$
\begin{gathered}
\frac{\partial u}{\partial t}+\frac{m_{1}}{m_{2}} \frac{\partial i}{\partial s}+m_{1} J=0 \\
\frac{\partial i}{\partial t}+m_{3} \frac{\partial u}{\partial s}+m_{4} i=0 \\
J=m_{2}\left(w+k_{1} u+k_{3} u^{3}\right) \\
\frac{1}{q_{0}} \frac{\partial w}{\partial t}=q_{1}+u
\end{gathered}
$$

где $i$ - ток, $m_{1}, m_{2}, m_{3}, m_{4}$ и $k_{1}, k_{3}, q_{0}, q_{1}$ - постоянные [ $\left.{ }^{3,4}\right]$.

Так как основная система теперь гиперболическая, удается вывести эволюционное уравнение, описывающее одну волну. При данных предпосылках оно имеет вид 


$$
\frac{\partial}{\partial \xi}\left(\frac{\partial u}{\partial s}+a_{1} u+\frac{1}{3} a_{3} u^{3}\right)=a_{4} u+a_{5},
$$

где $\xi=c_{0} t-s, a_{1}, a_{3}, a_{4}, a_{5}$ - постоянные. Здесь $c_{0}-$ скорость, определенная из телеграфных уравнений. X. M. Либерштейном показано $\left[{ }^{14}\right]$, что при разумно выбранных физических параметрах эта скорость действительно одного порядка со скоростью $c$ постоянного импульса, но в общем случае $c_{0} \neq c$. Считая амплитуду импульса $z$ от потенциала равновесия $\left[{ }^{1-5}\right]$, получим в конечном итоге эволюционное уравнение

$$
\begin{gathered}
\frac{\partial^{2} z}{\partial \xi \partial s}+f(z) \frac{\partial z}{\partial \xi}+g(z)=0 \\
f(z)=b_{0}+b_{1} z+b_{2} z^{2}, \quad g(z)=b_{00} z,
\end{gathered}
$$

где $b_{0}, b_{1}, b_{2}, b_{00}$ - постоянные. Детально вывод уравнения (2.6) приведен в $[4,15]$. Взамен уравнения третьего порядка (1.4) здесь получено уравнение второго порядка (2.6) в движущейся системе отсчета. Импульс постоянного профиля описывается теперь уравнением

$$
z^{\prime \prime}+f(z) z^{\prime}+\theta^{-1} g(z)=0,
$$

где $(\ldots)^{\prime}=\frac{\partial}{\partial \eta}, \eta=s+\theta \xi$. Параметр $\theta$ имеет здесь смысл псевдоскорости, которая позволяет перейти от скорости $c_{0}$ к действительной скорости $c$ импульса постоянного профиля

$$
c=\theta c_{0}(\theta-1)^{-1} \text {. }
$$

Легко установить, что в данном случае независимая переменная в уравнении (2.8) определяется выражением

$$
\eta=\theta c_{0}(t-s / c)
$$

Согласно данным Х. M. Либерштейна $\left[{ }^{14}\right]$, есть основание полагать, что $\theta>1$. Это означает, что $c>\mathrm{c}_{0}$, и если, согласно $\left[{ }^{14}\right]$, принимать $c_{0}=$ $=1,231 \mathrm{~cm} \cdot \mathrm{Mc}^{-1}$, то значение $\theta=2,6$ дает физически правильный результат $c=2 \mathrm{~cm} \cdot \mathrm{Mc}^{-1}$.

Уравнение постоянного профиля (2.8), вытекающее из эволюционного уравнения (2.6), совпадает точно с уравнением постоянного профиля (1.7) модели ФХН, если в последнем пренебречь третьей производной $\beta z^{\prime \prime \prime}$. Следует отметить, что как эволюционное уравнение (2.6), так и уравнение постоянного профиля (2.8) описывает процесс физически правильно. Об этом свидетельствуют результаты численного эксперимента $[4,15,16]$. Из них следует наличие порога, возможность усиления начального возбуждения и наличие стационарного профиля характерной формы с рефрактивной частью. Типичный импульс показан на рис. 2. При этом эволюционное уравнение решено псевдоспектральным методом, уравнение постоянного профиля - стандартным методом Рунге-Кутта. Проблем, связанных с неустойчивостью решений, не было.

Отметим, что в данном случае модельное уравнение (2.8) изучается в диапазоне параметров, где оба корня $z_{1}$ и $z_{2}$ квадратного уравнения $f(z)=b_{2} z^{2}+b_{1} z+b_{0}=0$ положительны: $z_{1}>0, z_{2}>0$. Этим оно отличается от хорошо известных моделей: в уравнении Ван дер Поля $z_{1}<0$, $z_{2}<0,\left|z_{1}\right|=z_{2}$; в уравнении лампового генератора с мягким режимом ([ $\left.{ }^{17}\right]$, с. 703) $z_{1}<0, z_{2}>0,\left|z_{1}\right| \neq z_{2}$. Качественный анализ уравнения (2.8) при $z_{1}>0, z_{2}>0$ будет представлен в следующей работе.

3. В случае модели ФХН неизвестная скорость $\Theta_{N}$ должна. иметь сверхвысокую точность. В модели, изложенной в разделе 2 , необходимо 


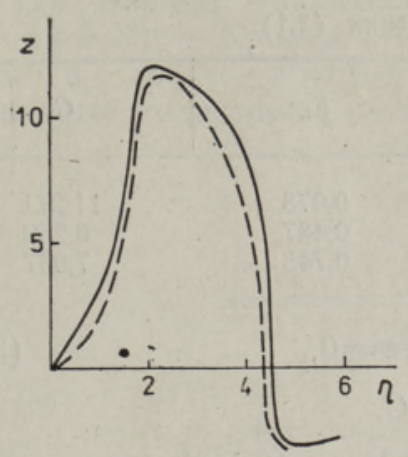

Рис. 2. Профиль импульса, определенный по уравнению (2.6) - прерывистая линия, по уравнению (2.8) - сплошная линия $\left[{ }^{15}\right]$.
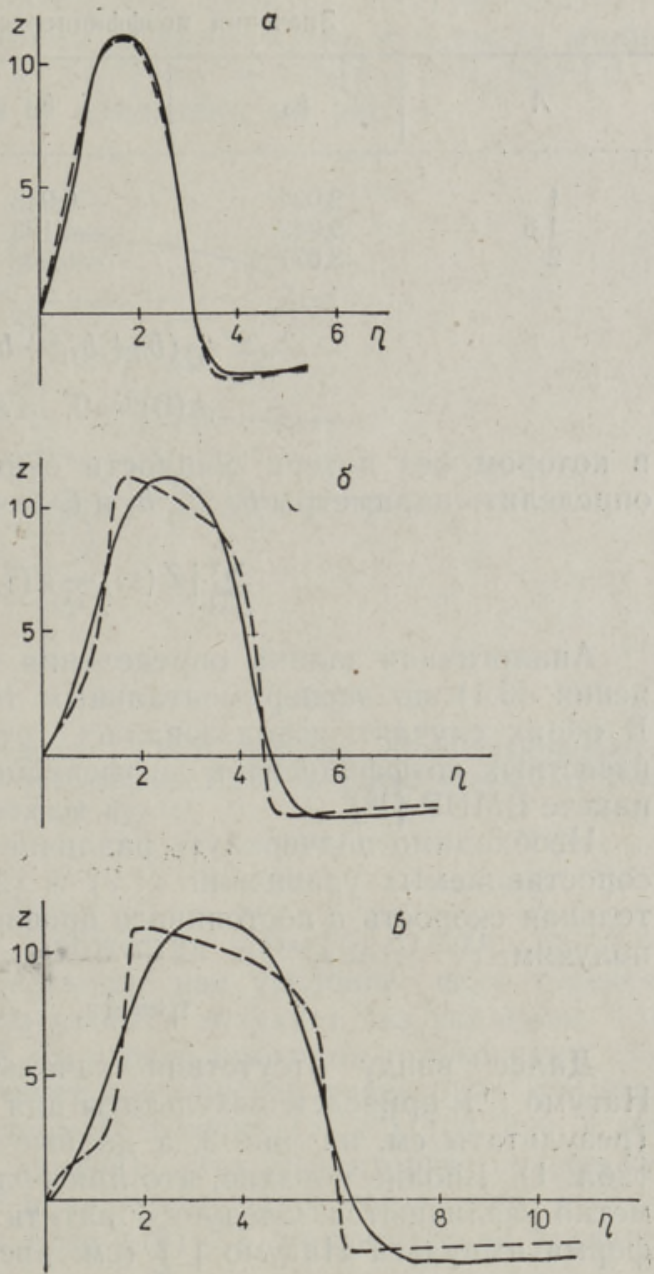

Рис. 3. Сопоставление профилей импульса, определенных по различным моделям: сплошная линия - по модели ФХН, прерывистая линия - по модели второго порядка. a $-A=1,0 ; \quad \delta=2,0$.

знать параметр $\Theta$ для корректировки скорости стационарного профиля (формула (2.9)). Ошибка или погрешность в определении $\Theta$ не меняет качественное поведение решения.

Допустим, что по модели ФХН удалось определить основную часть импульса (см. рис. 1). Возникает следующий вопрос: какими должны быть параметры модели (2.8), чтобы получить такой же результат количественно. Напомним, что результаты численного эксперимента $\left[{ }^{15,16}\right]$ показывали качественное совпадение форм обоих импульсов. Проблемы определения коэффициентов уравнения постоянного профиля (2.8) по известному экспериментальному профилю можно решить при помощи нелинейной регрессии $\left[{ }^{18}\right]$. Применим этот подход и в данном случае, считая профиль, определенный по модели ФХН, «экспериментальным».

За основу принято решение уравнения (1.7) (которое здесь обозначается как z) при параметрах $\beta=0,44262523060, \mu=3, \varepsilon=0,1$. Начальные условия определены изложенным в [ $\left.{ }^{1}\right]$ методом. Так как $Z(\tau)$ описывает распространение возбуждения начиная с $\tau \simeq 1$, то проведем замену времени $\tilde{\tau}=\tau-1$ и рассмотрим $Z(\tilde{\tau})$ на отрезке $\tilde{\tau} \in[0,5]$. В дальнейшем тильду опустим. Этот отрезок разделим точками $\tau_{i}$ на $N=50$ равных частей и вычислим значения $Z\left(\tau_{i}\right), i=1,2, \ldots, 50$.

Перепишем уравнение (2.8) в виде 


\begin{tabular}{l|c|c|c|c}
\hline$A$ & $b_{0}$ & $b_{1}$ & $b_{2}$ & $C$ \\
\hline 1,5 & 2,034 & $-0,945$ & 0,078 & 11,271 \\
2 & 5,049 & $-4,193$ & 0,437 & 6,259 \\
& 8,077 & $-6,948$ & 0,748 & 7,037 \\
& $z^{\prime \prime}+\left(b_{0}+b_{1} z+b_{2} z^{2}\right) z^{\prime}+z=0$, \\
$z(0)=0, \quad z^{\prime}(0)=C$,
\end{tabular}

в котором без потери общности считаем $\Theta^{-1} b_{00}=1$. Поставим задачу определить параметры $b_{0}, b_{1}, b_{2}$ и $C$ так, чтобы

$$
\sum_{i=1}^{N}\left[Z\left(\tau_{i}\right)-z\left(\tau_{i}\right)\right]^{2} \rightarrow \min
$$

Аналогичная задача определения неизвестных коэффициентов уравнения (3.1) по экспериментальным точкам была решена ранее [ $\left.{ }^{18}\right]$. В обоих случаях использовалась готовая программа определения неизвестных коэффициентов дифференциальных уравнений, имеющаяся в пакете BMDP $\left[{ }^{19}\right]$.

Необходимо подчеркнуть различие в независимых переменных $\tau$ и $\eta$ сопоставляемых уравнений (1.7) и (2.8). Если положить, что действительная скорость $c$ постоянного профиля в обоих случаях одинакова, то получим

$$
\eta=A \tau, \quad A=\Theta c_{0} .
$$

Далее, ввиду отсутствия начальных численных данных модели Нагумо $\left[{ }^{1}\right]$, приведем результаты для трех значений $A=1 ; 1,5 ; 2 \mathrm{~cm} \cdot \mathrm{c}^{-1}$ (результаты см. на рис. 3 , а данные параметров $b_{0}, b_{1}, b_{2}$ и $C-$ в табл. 1). Вполне понятно, что при больших значениях А результаты заметно различаются. Следует обратить внимание на то, что качественная форма импульса Нагумо [ $\left.{ }^{1}\right]$ (см. рис. 1) не вполне соответствует экспериментально определенной форме импульса, основная часть которой имеет несимметричный вид $\left[{ }^{2,5,7}\right]$, похожий на изображенный на рис. 2 профиль.

4. Харақтерным свойством поведения реального нервного импульса является наличие порогового эффекта, т. е. при одних начальных условиях импульс сразу затухает, а при других - происходит усиление, которое затем заменяется затуханием. Рассмотрим, каким образом это свойство отражается в решении уравнения (2.8).

Перепишем уравнение (2.8) с начальными условиями $z(0)=0$, $z^{\prime}(0)=C$ в виде системы

$$
\begin{aligned}
& \frac{d z}{d t}=y, \\
& \frac{d y}{d t}=-\left(b_{0}+b_{1} z+b_{2} z^{2}\right) y-b_{3} z \\
& z(0)=0, \quad y(0)=C,
\end{aligned}
$$

где $b_{3}=\theta^{-1}>0$. По-прежнему считаем, что корни $z_{1}$ и $z_{2}$ уравнения $b_{2} z^{2}+b_{1} z+b_{0}=0$ положительны.

На фазовой плоскости ' $(y, z)$ решение системы (4.1) удовлетворяет следующему дифференциальному уравнению:

$$
\frac{d u}{d z}=-\left(b_{0}+b_{1} z+b_{2} z^{2}\right)-\frac{b_{3} z}{y},
$$


откуда найдем уравнение нуль-изоклины $y=h(z): h(z)=-b_{3} z\left(b_{0}+\right.$ $\left.+b_{1} z+b_{2} z^{2}\right)^{-1}$. Кривая $y=h(z)$ имеет в промежутке $z_{1}<z<z_{2}$ минимум в точке $z^{*}=\left(b_{0} / b_{2}\right)^{1 / 2}$ и $h\left(z^{*}\right)=y^{*}=-b_{3}\left[b_{1}+2\left(b_{0} / b_{2}\right)^{1 / 2}\right]^{-1}$. Типичные траектории системы (4.1) приведены на рис. 4.

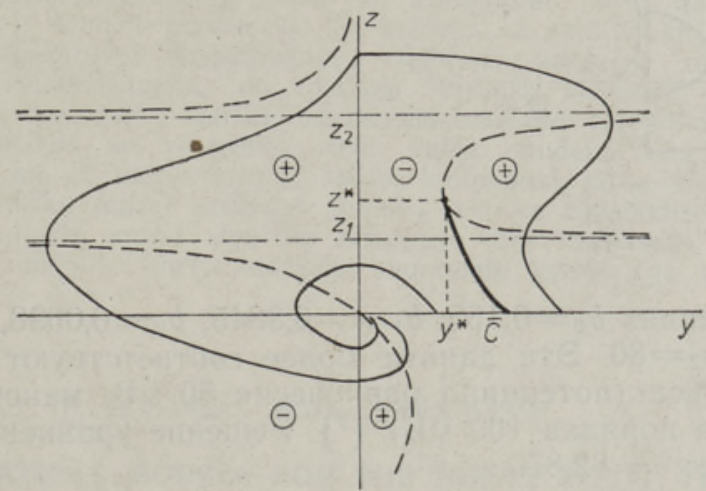

Рнс. 4. Фазовая плоскость. Прерывистые линии - нуль-изоклины, штрих-пунктирные асимптоты к ним, сплошные линии - траекторни. Плюс и минус указывают знак производной $d y / d z$.

Если траектория достигает множества $D=\{(y, z) \in D: y>h(z)$, $\left.z_{1}<z<z_{2}\right\}$, то происходит интересующее нас усиление; если траектория множества $D$ не достигает, то процесс затухает без усиления. Так как через каждую точку фазовой плоскости проходит только одна траектория системы (4.1), то существует критическая траектория $y=\bar{y}(z)$, которая соприкасается с множеством $D$ в точке $\left(y^{*}, z^{*}\right)$ (на рис. 4 эта траектория обозначена жирной линией). Эта траектория пересекает ось $y$ в точке $(\bar{c}, 0)$. Величина $\bar{c}$ и является пороговым; если $y(0)=$ $=C>\bar{c}$, то происходит усиление, если $y(0)<\bar{c}$, то процесс не усиливается.

Пороговое значение $\bar{c}$ легко определить, если решать уравнение (4.2) при начальном значении $y\left(z^{*}\right)=y^{*}$ и вычислять $\bar{c}=y(0)$. Уравнение (4.2) является уравнением Абеля $\left(\left[{ }^{20}\right]\right.$, c. 26). Для численных расчетов уместно привести его к привычному виду с положительным направлением независимой переменной $z$. Произведя замену $x=\left(b_{0} / b_{2}\right)^{1 / 2}-z$, получим

$$
\begin{aligned}
& \frac{d y}{d x}=\left(c_{0}-c_{1} x+c_{2} x^{2}\right)+b_{3} \frac{\left(b_{0} / b_{2}\right)^{1 / 2}-x}{y}, \\
& y(0)=-\frac{b_{3}}{c_{1}}
\end{aligned}
$$

где $c_{0}=2 b_{0}+b_{1}\left(b_{0} / b_{2}\right)^{1 / 2}, \quad c_{1}=b_{1}+2\left(b_{0} b_{2}\right)^{1 / 2}, c_{2}=b_{2}$.

Пороговое значение $\bar{c}$ определяется как

$$
\bar{c}=y\left[\left(b_{0} / b_{2}\right)^{1 / 2}\right] .
$$

Приведем два примера.

1. Пусть $b_{0}=3, b_{1}=-3, b_{2}=0,3, b_{3}=1$, тогда $z_{1}=1,127, z_{2}=8,873$. Численное решение уравнения (4.3) методом Рунге-Кутта с шагом $\Delta x=0,002$ дает $\bar{c}=3,367$. На рис. 5 показаны фазовые траектории при $\bar{c}=3,365$ и $\bar{c}=3,369$, отличающиеся существенно, 


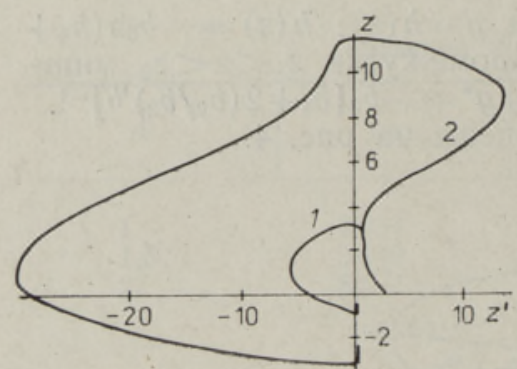

Рис. 5. Фазовая плоскость для примера 1. Кривая $1-\bar{c}=3,365$, кривая $2-$ $\bar{c}=3,369$.

2. При значениях $b_{0}=6,155, b_{1}=-0,3845, b_{2}=0,0038, b_{3}=1\left[{ }^{15}\right]$ получим $z_{1}=20, z_{2}=80$. Эти данные более соответствуют реальным параметрам импульса (потенциал равновесия 50 мВ, максимальная амплитуда импульса порядка 100 мВ) [7]. Решение уравнения (4.3) с шагом $\Delta x=0,02$ дает $\bar{c}=82,876$.

5. Кроме прямого моделирования процесса в нервном волокне эволюционное уравнение (2.6) или его стационарная форма (2.8) могут служить модельными в задачах сформирования импульсов заданного профиля в активных средах.

Авторы благодарны А. Уус за численные примеры определения порога.

\section{ЛИТЕ РАТ У РА}

1. Nagumo, J., Arimoto, S., Yoshizawa, S. Proc. IRE, 50, 2061-2070 (1962).

2. Scott, A. C. Neurophysics. New York, Wiley, 1977.

3. Engelbrecht, J. Proc. Roy. Soc. London, A375, 195-209 (1981).

4. Энгельбрехт Ю. К. Биофизнка, 30, вып. 1, 124-128 (1985).

5. Hodgkin, A. L. The Conduction of the Nervous Impulse. Liverpool University, 1964.

6. Васильев В. А., Романовский Ю. М., Яхно В. Г. Усп. физ. наук, 128, вып. 4, 625666 (1979).

7. Marsocci, V. A. CRC Crit. Rev. in Biomed. Engng., 8(2), 135-194 (1982).

8. Зыков В. С. Моделирование волновых процессов в возбудимых средах. М., «Наука», 1984.

9. Rinzel, J., Keller, J. B. Biophys. J., 13, 1313-1337 (1973).

10. Hastings, S. P. SIAM J. Appl. Math., 42, № 2, 247-260 (1982).

11. Feroe, J. A. SIAM J. Appl. Math., 42, № 2, 235-246 (1982).

12. Jones, C. K. R. T. Contemporary Math., 17, 287-292 (1983).

13. Hénon, M. Comm. Math. Phys., 50, 69-77 (1976) - перевод в сб. Странные аттракторы. М., «Мир», 1981, 152-163.

14. Lieberstein, H. M. Math. Biosci., 1, 45-69 (1967).

15. Engelbrecht, J. Wave Motion, 8, 93-100 (1986).

16. Энгельбрехт Ю. К. В кн.: Вопросы нелинейной механики сплошной среды. Таллин, «Валгус», 1985, 119-131.

17. Андронов А. А., Витт А. А., Хайкин С. Э. Теория колебаний, 2-е изд. М., Гос. нзд. фнз.-мат., 1959.

18. Энгельбрехт Ю., Тобиас T. Изв. АН ЭССР. Физ. Матем., 34, № 2, 154-157 (1985).

19. BMDP, Biomedical Computer Programs. Ed. by Dixon, W. University of California Press, 1979.

20. Kamke E. Differentialgleichungen. Lösungsmethoden und Lösungen. I. Leipzig, Akademische Verlagsgesellschaft, 1967. 


\section{NARVIIMPULSI LEVIMISE MATEMAATILISTEST MUDELITEST}

Impulsi levimist närvikius on tavaliselt kirjeldatud FitzHugh-Nagumo mudelíga, mille järgi impulsi statsionaarne profiil on kolmandat järku hariliku diferentsiaalvõrrandi lahend [ $\left.{ }^{1}\right]$. Mudeli puuduseks on asjaolu, et profiili kirjeldamiseks pikemal ajalōigul tuleb füüsikalisest eksperimendist leitavad kordajad määrata ebareaalselt suure täpsusega. Teine võimalus on tuletada levimist kirjeldav evolutsioonivõrrand ja sellest lähtudes teist järku harilik diferentsiaalvõrrand statsionaarse profiili kirjeldamiseks $\left[{ }^{3,4}\right]$. Artiklis on võrreldud neid kahte mudelit ning leitud teist järku võrrandi kordajad nii, et vastav profiil sobiks kolmandat järku võ̃randist leituga, ja näidatud, et teist järku mudel kirjeldab impulsi levi ka füüsikalise täpsusega määratud kordajate puhul. On antud algoritm niisuguse läve leidmiseks, millest väiksemate algväärtuste puhul impulss kiirelt sumbub, suuremate korral aga võimendub.

\section{J. ENGELBRECHT, $\overline{|T . T O B I A S|}$, Marju KAARMANN}

\section{ON MATHEMATICAL MODELS FOR THE NERVE PULSE TRANSMISSION}

The well-known FitzHugh-Nagumo model for the nerve pulse transmission [ $\left.{ }^{1}\right]$ describes the stationary profile as the solution to the third order nonlinear differential equation (1.7). In numerical calculations the velocity of the nerve pulse must be determined with rather great accuracy in order to calculate the slow motion towards the equilibrium. The evolution equation derived for the pulse transmission $[3,4]$ leads to the second order stationary profile equation $(2.8)$. This equation belongs to the class of the Liénard equations. The comparative analysis of these two models is presented. The coefficients of the second order stationary profile equations are determined by matching the pulse profile with the solution of the third-order stationary profile equation corresponding to the FitzHugh-Nagumo model. The respective inverse problem is solved by using the standard Biomedical Computer Programs, [19]. The second order stationary profile equation derived from the corresponding evolution equation permits to describe the important physical phenomena of transmission including the existence of the threshold. The value of the threshold is here connecfed with the initial condition. A simple algorithm is proposed for determining this critical initial (threshold) value. Every profile with the initial condition above the threshold value corresponds to the amplification, 